\title{
Efficacy of Perioperative Intercostal Analgesia via a Multimodal Analgesic Regimen for Chronic Post-Thoracotomy Pain During Postoperative Follow-Up: A Big-Data, Intelligence Platform-Based Analysis
}

\author{
Yijin Yu (D) \\ Lingyan Cui' \\ Lu Qian (1D' \\ Min Lei' \\ Qi Bao' \\ Qingxin Zeng ${ }^{2}$ \\ Zhao Chen ${ }^{2}$ \\ Shaohua $\mathrm{Xu}^{2}$ \\ Junran Xie'
}

'Department of Anesthesiology, Sir Run Run Shaw Hospital, School of Medicine, Zhejiang University, Hangzhou, Zhejiang, People's Republic of China; ${ }^{2}$ Department of Thoracic Surgery, Sir Run Run Shaw Hospital, School of Medicine, Zhejiang University, Hangzhou, Zhejiang, People's Republic of China
Correspondence: Junran Xie

Department of Anesthesiology, Sir Run Run Shaw Hospital, School of Medicine,

Zhejiang University, 3 Qingchun Road

East, Jianggan District, Hangzhou,

Zhejiang, People's Republic of China

Tel +86 I3858126216

Email xiejunran@zju.edu.cn
Background: Chronic post-thoracotomy pain is still an obstacle for lung-cancer patients even after less invasive surgical procedures. It is unclear whether intercostal analgesia is as useful in the prevention of postoperative chronic pain as it is for acute pain for video-assisted thoracoscopic surgery (VATS). The purpose of this study was to evaluate the efficacy of perioperative intercostal analgesia for chronic pain via a multimodal analgesic regimen for VATS during 6 months of postoperative follow-up.

Methods: We identified 837 cases of VATS from August 2016 to August 2018. Patients were treated by perioperative intercostal analgesia with $0.75 \%$ ropivacaine $50 \mathrm{mg}$ through the intercostal catheter every 8 hours until chest tube extubation (INA group) or conventional analgesia with preoperative $0.75 \%$ ropivacaine $50 \mathrm{mg}$ at incision once (CON group). Numerical rating scale (NRS) and neuropathic pain were evaluated in 6 months of postsurgery follow-up. Postoperative adverse effects were recorded.

Results: In total, there were 419 patients in INA group and 418 patients in CON group. Scores of NRS with motion was lower in INA group at 3 postoperative days $(P=0.032)$. Occurrence of chronic pain was $28.4 \%$ in INA group and $32.8 \%$ in CON group at 6 postoperative months, $10.6 \%$ of patients experienced increasing pain from 3 to 6 months. Occurrence of considerable neuropathic pain (ID pain score $\geq 2$ ) was $2.1 \%$ in INA group and $3.1 \%$ in CON group at 6 postoperative months. No differences were found between the two groups. Occurrence of numbness was lower in INA group $(6.7 \%$ vs $10.5 \%, P=0.031)$, and other pain symptoms did not differ between the groups. The incidence of dizziness, nausea, vomiting and atelectasis was not different between the two groups.

Conclusion: In a multimodal analgesic regimen of VATS, perioperative intercostal analgesia with $0.75 \%$ ropivacaine infusion $50 \mathrm{mg}$ three times in a day does not have an obvious effect on chronic post-thoracotomy pain.

Keywords: chronic pain, neuropathic pain, intercostal analgesia, video-assisted thoracoscopic surgery

\section{Introduction}

Patients often experience acute and chronic pain after thoracotomy. Bayman et al. reported a greater pain during the first 3 postoperative days, which was associated with the higher chance of chronic pain. The incidence of persistent pain was $27 \%$ in 
thoracotomy, and pain limited the daily activities in $8.2 \%$ of the patients. The incidence of persistent pain in videoassisted thoracoscopic surgery (VATS) was similar to thoracotomy. ${ }^{1}$ Homma et al. reported that $25.9 \%$ who underwent VATS experienced postoperative neuropathic pain, and $18.8 \%$ experienced pain that persisted one year postoperatively. $^{2}$

Medications, such as antidepressants, nonsteroidal antiinflammatory drugs and anticonvulsants are not always effective in preventing persistent pain. ${ }^{3}$ Regional anesthesia such as stellate ganglion blocks, epidural blocks and intercostal nerve blocks may have some beneficial effects, ${ }^{4}$ although analgesic effects of these blocks with local anesthetics are often short-acting. ${ }^{4}$ A recently published case report suggested that local anesthetics with intercostal nerve blocks in high concentrations (5\% tetracaine) provide longer sensory blockade and are effective for preventing chronic pain syndromes. ${ }^{5}$ Also some studies showed that regional anesthesia could prevent chronic pain of thoracotomy. Compared with fentanyl iv for 48 hours, epidural morphine and ropivacaine reduced pain at 3 and 4 postoperative months. ${ }^{6}$ Compared with cryoanalgesia of intercostal nerves, epidural bupivacaine and morphine for 72 hours reduced chronic allodynia at 6 and 12 postoperative months. ${ }^{7}$ The incidence of chronic pain was $45 \%$ after receiving epidural bupivacaine and morphine for 2 days, and the incidence of chronic pain was $78 \%$ after receiving morphine iv at 6 postoperative months. ${ }^{8}$

Thoracic epidural analgesia is effective in thoracotomy patients, but epidural analgesia may result in epidural hematoma, dural perforation, and urinary retention., ${ }^{9,10}$ Intercostal analgesia with a single intercostal catheter could avoid such complications. ${ }^{11}$ In a review, using thoracic epidural anaesthesia in five of seven included studies showed the strongest effect, but whether other interventions such as intercostal analgesia have the same effect is unknown. ${ }^{12}$ We hypothesized perioperative intercostal analgesia may be useful to prevent chronic neuropathic pain.

It was not known whether neuropathic pain recovers more slowly or occurs less frequently than nonneuropathic pain following thoracotomy, and chronic pain diagnosed as neuropathic makes it difficult to be sure. Questionnaires are being developed to try to identify the qualities of neuropathic pain. ${ }^{13,14}$ At present, the scales of LANSS, NPQ and ID Pain are commonly used with high sensitivity and specificity. The accuracy, specificity, sensitivity, positive and negative predictive value of three scales have been compared in a study. ${ }^{15}$ Area under ROC curve showed that accuracy of ID Pain and LANSS were better. Specificity and sensitivity of LANSS $\geq 12$ were $97.1 \%$ and $80 \%$, specificity and sensitivity of ID Pain $\geq 2$ were $90.0 \%$ and $87.1 \%$. These two scales were both relatively ideal. The scale of LANSS was evaluated by doctors and diagnosed by measuring acupuncture threshold which increases difficulty during postoperative follow-up. The scale of ID Pain was a self-evaluated scale. It was easier and more suitable for postoperative followup. ID Pain score $\geq 2$ should be diagnosed as considerable neuropathic pain, and ID Pain score $\geq 1$ should be diagnosed as possible neuropathic pain. In this study, we evaluated the effect of perioperative intercostal analgesia on chronic postoperative pain and neuropathic pain during VATS in 6 postoperative months follow-up.

\section{Methods \\ Study Design and Patients}

This study was approved by the Institutional Review Board (approval number: 20180604-7) on June 4, 2018. We identified 2012 patients who underwent VATS on the intelligence database platform (Yidu Cloud Technology Ltd, Beijing, China) at Department of Thoracic Surgery, Sir Run Run Shaw Hospital, School of Medicine, Zhejiang University between August 2016 and August 2018. Patient information was obtained from data base platform. Inclusion criteria: elective lobectomy or segmentectomy under VATS between 18 and 80 years old. Exclusion criteria were as follows: secondary surgery, history of opioid use, preoperative chronic pain, conversion to a thoracotomy, serious preoperative and postoperative complications. There were two groups, one group accepted perioperative intercostal analgesia (INA group) and another group accepted conventional analgesia (CON group). All patients provided written informed consent between August 2016 and August 2018.

\section{Procedure of Anesthesia}

After patient monitoring, the induction of anesthesia was an intravenous bolus administration of $0.3-0.4 \mathrm{ug} / \mathrm{kg}$ sufentanil, $1.5-2 \mathrm{mg} / \mathrm{kg}$ propofol, and $0.2 \mathrm{mg} / \mathrm{kg}$ cisatracurium besylate. After endotracheal intubation with double-lumen endotracheal tube, general anesthesia was maintained with 1-2\% sevoflurane, 4-6 mg/ $\mathrm{kg} / \mathrm{h}$ propofol, and $15-20 \mathrm{ug} / \mathrm{kg} /$ $\mathrm{h}$ remifentanil. The bispectral index was monitored and maintained within the range of 40-60. Mechanical 
ventilation was titrated to achieve the end-tidal carbon dioxide concentration between 35 and $40 \mathrm{mmHg}$, one-lung ventilation was maintained when segmentectomy was performed.

\section{Surgical Technique}

The patient was placed in a standard lateral position; at the anterior axillary line and fourth or fifth intercostal space, a 3-cm incision was performed. At the midaxillary line and seventh or eighth intercostal space, another $0.5 \mathrm{~cm}$ observation hole was created, and at the next rib and posterior axillary line, a $1 \mathrm{~cm}$ supplementary hole was created. When the lesion was located at the upper lobe of the lung, the fourth intercostal space was chosen. Rib resection or spreading was not performed in this study. Further, a thoracic drainage tube was inserted at the seventh or eighth intercostal space. This procedure was performed by skilled attendants in the same medical group.

\section{Pain Management}

At the end of surgery, a 5-Fr 20-cm 16G radio-opaque polyurethane catheter (Biosensors International, CV-50120YT) was inserted under a thoracoscope and $50 \mathrm{mg}$ of $0.75 \%$ ropivacaine was injected in the intercostal space. The intercostal catheter was placed in the subpleural space at the same level where the chest tube was placed. When the patients were sent to the general ward, local anesthetic infusion of $0.75 \%$ ropivacaine $50 \mathrm{mg}$ was administered every 8 hours (q8h) through the subpleural catheters (Figure 1). After the surgery, the infusion continued until the chest tube was removed. In addition, intraoperative analgesic drugs consisting of dezocine $5 \mathrm{mg}$, parecoxib
A

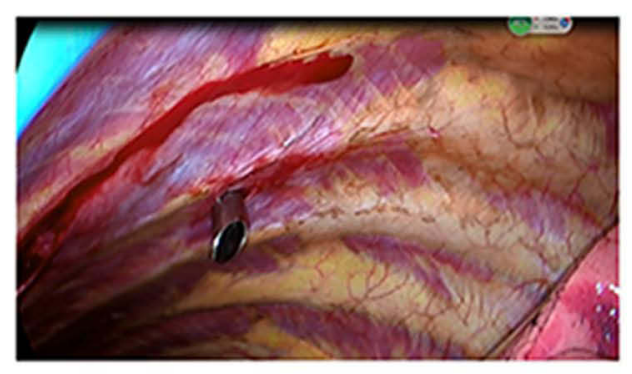

C

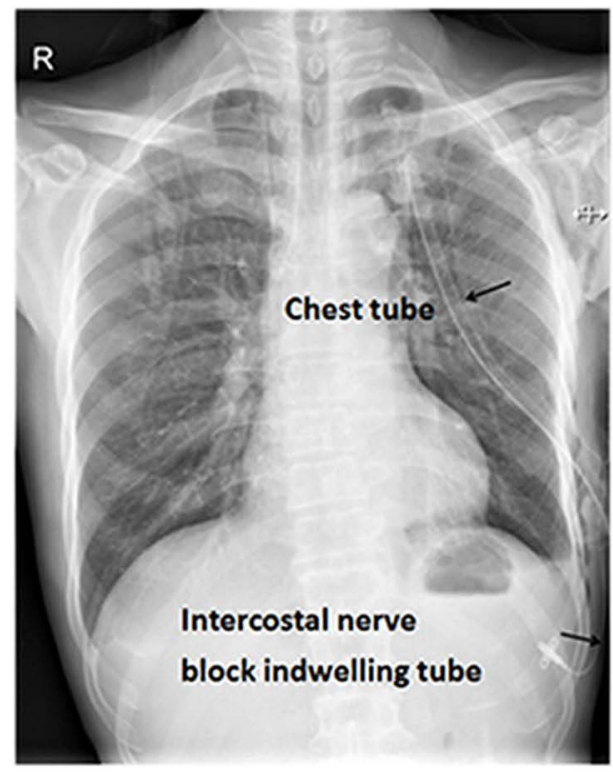

B

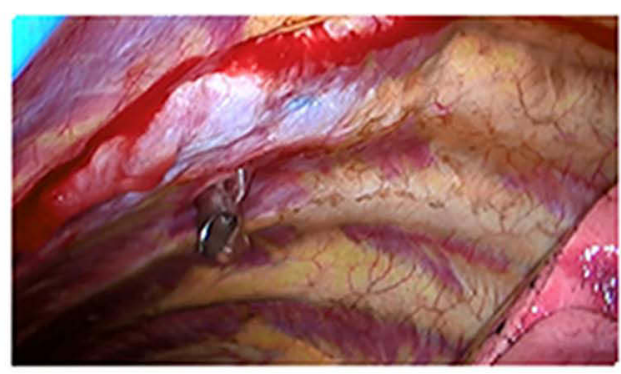

D

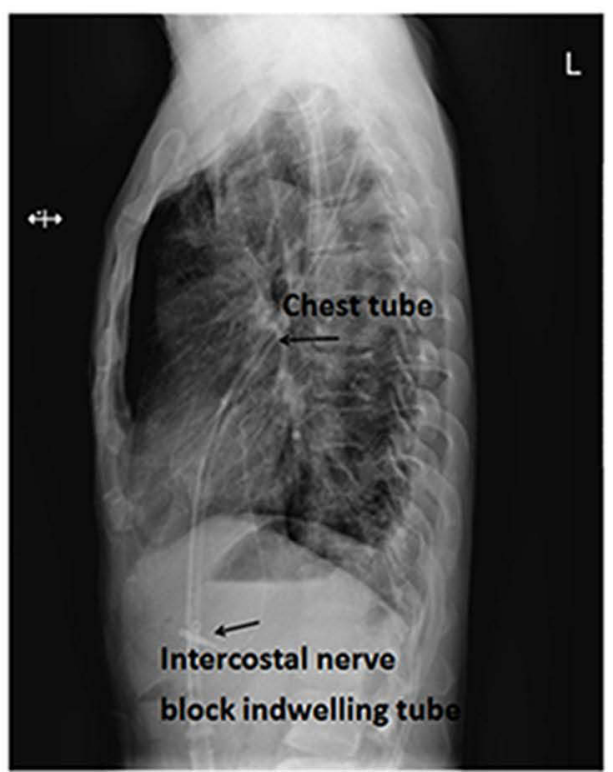

Figure I Intercostal catheter was placed in the sub-pleural space, which was the same intercostal space of chest tube. (A) Intercostal catheter placement, (B) Intercostal nerve analgesic administration, (C) Frontal chest radiograph, (D) Lateral chest radiograph. 
$40 \mathrm{mg}$ and postoperative oral medication consisting of paracetamol and dihydrocodeine tartrate tablets (acetaminophen $500 \mathrm{mg}$ and dihydrocodeine tartrate $10 \mathrm{mg}$ ) $500 \mathrm{mg}$ q $8 \mathrm{~h}$ and parecoxib $40 \mathrm{mg}$ q12h were administered to the patients until discharge from hospital.

\section{Clinical Endpoints}

The first endpoint was numeric rating scale (NRS) scores at different follow-up durations. Patient's pain was assessed by the NRS ( $0=$ "no pain", $10=$ "worst possible pain"). The pain degrees based on the NRS scores were defined as follows: none $=0$, mild $=1-3$, moderate $=4-6$, and severe $=7-10$. The pain score was recorded.

Other endpoints included the proportion of patients who developed chronic pain and neuropathic pain during 6 postoperative months. We defined symptoms of neuropathic pain as pins and needles, burning, numbness, electricity and touch aggravation. ${ }^{16}$ Neuropathic pain was evaluated by ID Pain questionnaire. ${ }^{17}$ The grading methods of neuropathic pain were as follows: $-1,0$ (unlikely); 1 (possible); 2, 3 (considerable); and 4, 5 (definite). Neuropathic pain was defined if the ID Pain score was $\geq 2$. The ID Pain score was evaluated by an anesthetist during 6 months follow-up over the telephone.

\section{Statistical Analysis}

Continuous data are described as mean \pm standard deviation or median (interquartile range). Inter-group comparison was performed by the independent-samples $t$-test for normally distributed data and the Mann-Whitney $U$-test for abnormally distributed data. Count data are expressed in percentage. The chi-square test or Fisher's exact test were used for inter-group comparison. Statistical significance was considered at $\mathrm{P}<0.05$. Calculations were completed with SPSS 22.

\section{Results}

Medical records of 2012 patients who underwent either INA or CON for VATS between August 2016 and August 2018 were reviewed. We excluded patients who met the exclusion criteria $(\mathrm{N}=276)$ : secondary surgery $(\mathrm{N}$ $=133)$, preoperative history of chronic pain $(\mathrm{N}=25)$, conversion to a thoracotomy $(\mathrm{N}=68)$, perioperative heavy bleeding $(\mathrm{N}=24)$, postoperative severe lung infection $(\mathrm{N}=15)$, history of cerebral infarction $(\mathrm{N}=4)$, hematoma of intercostal puncture $(\mathrm{N}=7)$. Postoperative follow-up loss for pain assessment were 418 in INA group and 481 in CON group. Thus, 837 patients were included
(419 in INA group and 418 in CON group) in this study (Figure 2). The general information of all the patients $(n=$ 837 ) is listed in Table 1.

\section{NRS Scores During 6 Postoperative Months Follow-Up}

The pain was felt along the VATs scar during 6 postoperative months follow-up. NRS scores of pain intensity varied from a minimum of 0 to a maximum of 4.0 in INA and CON groups at the time of extubation in PACU and 3 postoperative days. Scores varied from $0-4$ in INA group at 1 postoperative month and $0-3$ at 3 and 6 postoperative months. Scores varied from $0-3$ in $\mathrm{CON}$ group at 1 and 3 postoperative months and $0-4$ at 6 postoperative months. There were no significant differences between the two groups (Table 2).

\section{Occurrence of Chronic Pain and Neuropathic Pain at 3 and 6 Postoperative Months}

Altogether $25.6 \%$ patients had chronic pain at 3 postoperative months, $23.6 \%$ in INA group and $27.5 \%$ in CON group $(P=0.198) .30 .6 \%$ patients had chronic pain at 6 postoperative months, $28.4 \%$ in INA group and $32.8 \%$ in CON group $(P=0.170)$ (Figure 3 ). $2.6 \%$ patients had considerable neuropathic pain (ID Pain $\geq 2$ ) at 6 postoperative months, $2.1 \%$ in INA group and $3.1 \%$ in CON group $(P=0.384) .12 .9 \%$ patients had possible neuropathic pain (ID Pain $\geq 1$ ) at 6 postoperative months, $12.4 \%$ in INA group and $13.4 \%$ in CON group $(P=0.670)$.

When assessed with the ID Pain questionnaire, neuropathic pain symptoms and signs were described from high to low as pins and needles (16.8\%), touch aggravation $(9.6 \%)$, numbness $(8.6 \%)$, electricity $(3.1 \%)$, and burning $(0.7 \%)$ at 6 postoperative months. Pins and needles (17.2\%), touch aggravation (9.3\%), numbness (6.7\%), electricity $(2.9 \%)$, and burning $(1.0 \%)$ were observed in the INA group, while pins and needles (16.5\%), touch aggravation $(9.8 \%)$, numbness $(10.5 \%)$, electricity $(3.3 \%)$, and burning $(0.5 \%)$ were observed in the CON group. The incidence of numbness was lower in the INA group $(6.7 \%$ vs $10.5 \%, P=0.031)$ than in the CON group.

Pain from the area outside the incision, such as near the breast, around the ribs along the line area, the arm, shoulder, and back, was experienced by $10.2 \%$ patients; $8.8 \%$ of these patients were found in the INA group and $11.5 \%$ were found in the CON group $(P=0.124)$. 


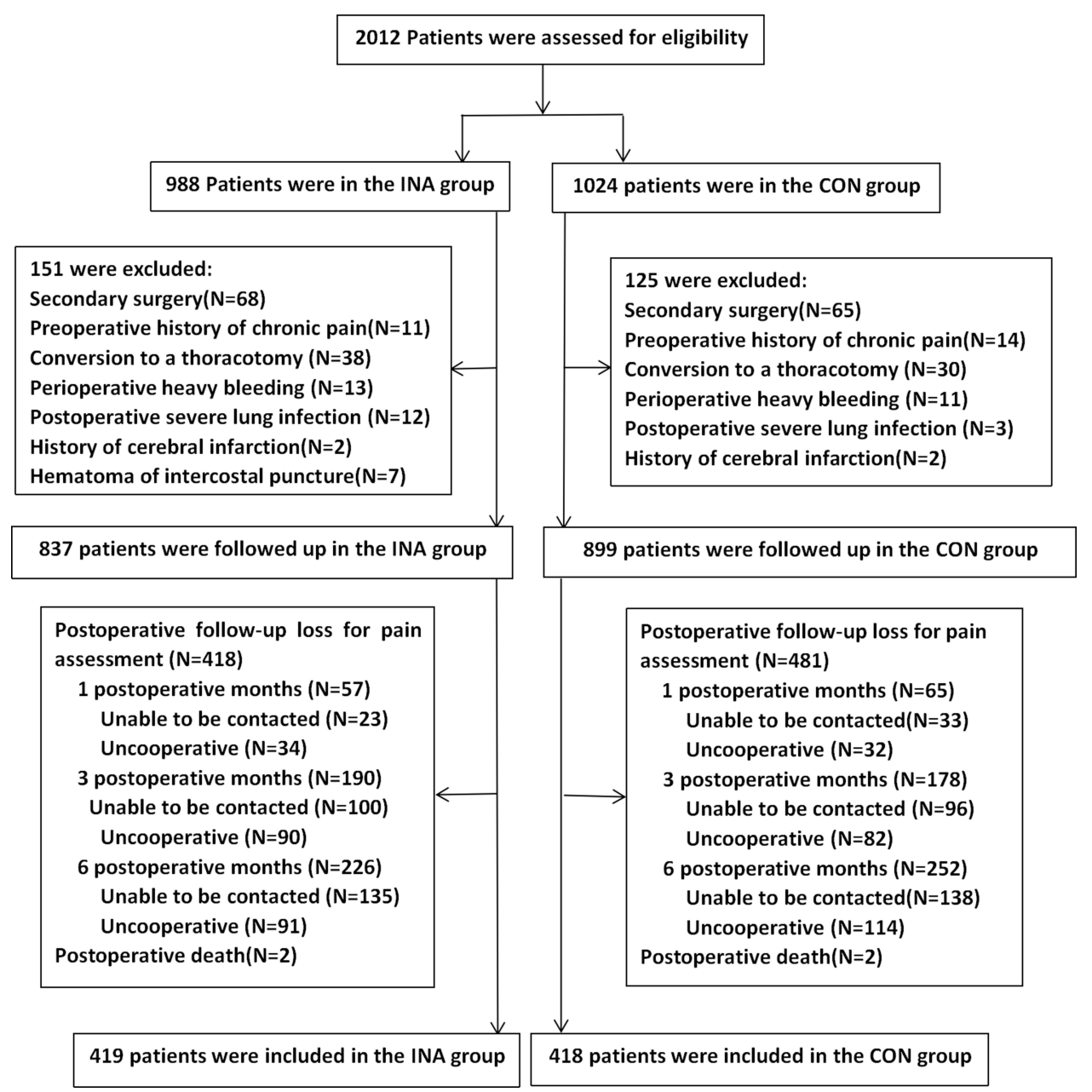

Figure 2 Study flow chart.

Pain aggravation from 3-6 postoperative months was experienced by $10.6 \%$ of patients; $9.3 \%$ of patients were in the INA group and $12.0 \%$ were in the CON group $(P=$ 0.129) (Figure 3).

\section{Postoperative Side Effects}

Blurred vision, arrhythmia, dyspnea and epilepsy were not found in the INA group and the CON group during postoperative follow-up. The incidence of dizziness, nausea and vomiting were not different between the INA group and the CON group (Table 3).

\section{Discussion}

In our study, because of the analgesic differences between the INA and CON groups, we expected to observe a difference in postoperative pain intensity between these two groups, and we found that pain intensity was lower in the INA group only at 3 postoperative days when patients were in an active state. Perioperative intercostal analgesia did not affect the incidence of chronic pain and neuropathic pain at 3 and 6 months after VATS, although we found a decrease of numbness in the INA group at 6 postoperative months. Further, no significant differences were found in the other variables between the two groups.

Chronic pain after thoracotomy is defined as pain persisting or recurring for $\geq 2$ months after operation. ${ }^{18}$ Extensive nerve injury often occurs with the use of a rib retractor which affects the function of the intercostal nerve by $50-100 \% .{ }^{19}$ VATs is expected to reduce pain due to mini-incisions. However, VATS may not reduce intercostal nerve damage, because the operation of inflexible trocars can cause nerve injury directly or indirectly. ${ }^{20}$ Homma et al. 
Table I Patient Characteristics

\begin{tabular}{|c|c|c|c|c|}
\hline & $\begin{array}{l}\text { INA Group } \\
(\mathrm{N}=419)\end{array}$ & $\begin{array}{l}\text { CON Group } \\
(N=4 \mid 8)\end{array}$ & $t / X^{2}$ & $p$ \\
\hline Male(n,\%) & $239(57.0)$ & $232(55.5)$ & 0.144 & 0.352 \\
\hline Age $(y)$ & $59.53 \pm 10.89$ & $60.95 \pm 10.19$ & 1.941 & 0.053 \\
\hline Height $(\mathrm{cm})$ & $163.09 \pm 6.99$ & $162.22 \pm 7.43$ & -1.759 & 0.079 \\
\hline Weight (cm) & $59.15 \pm 6.58$ & $60.18 \pm 9.39$ & 1.846 & 0.065 \\
\hline Tumor size $\left(\mathrm{cm}^{2}\right)$ & $1.58 \pm 0.67$ & $1.53 \pm 0.70$ & -0.979 & 0.328 \\
\hline Lymph node dissection (n,\%) & $229(54.7)$ & $220(52.6)$ & 0.268 & 0.580 \\
\hline Duration of anesthesia (min) & $123.13 \pm 37.18$ & $119.65 \pm 31.42$ & -1.460 & 0.145 \\
\hline Duration of operation (min) & $91.97 \pm 37.69$ & $87.40 \pm 36.27$ & -1.785 & 0.075 \\
\hline Duration of chest tube drainage (d) & $3.52 \pm 1.55$ & $3.55 \pm 2.13$ & 0.214 & 0.830 \\
\hline Postoperative duration of stay (d) & $4.16 \pm 1.59$ & $3.92 \pm 2.17$ & -1.797 & 0.073 \\
\hline Intraoperative usage of sufentanil (ug) & $25.47 \pm 19.92$ & $22.95 \pm 18.88$ & -1.872 & 0.062 \\
\hline Intraoperative usage of dezocine $(\mathrm{mg})$ & $7.55 \pm 2.98$ & $7.89 \pm 3.17$ & 1.615 & 0.107 \\
\hline Perioperative usage of parecoxib $(\mathrm{mg})$ & $322.24 \pm 110.52$ & $306.79 \pm 141.99$ & -1.756 & 0.079 \\
\hline Perioperative usage of paracetamol and dihydrocodeine tartrate tablets $(\mathrm{mg})$ & $6035.80 \pm 2046.10$ & $5824.16 \pm 3038.65$ & -1.182 & 0.237 \\
\hline Postoperative atelectasis $(\mathrm{n}, \%)$ & $364(86.9)$ & 357(85.4) & 0.377 & 0.304 \\
\hline
\end{tabular}

Notes: The data are presented as the means \pm standard deviation (SD), or numbers (\%); there were no significant differences between groups, as determined by the independent-samples $t$-test, the Chi-square test $(P>0.05)$.

Table 2 Numerical Rating Scales Scores of Pain Intensity (Median, Interquartile Range)

\begin{tabular}{|c|c|c|c|c|}
\hline & $\begin{array}{c}\text { INA } \\
(N=419)\end{array}$ & $\begin{array}{c}\text { CON } \\
(N=418)\end{array}$ & $\mathbf{Z}$ & $\boldsymbol{P}$ \\
\hline Extubation in PACU (at rest) & $0(0,2)$ & $0(0,2)$ & -0.240 & 0.810 \\
\hline Extubation in PACU (with motion) & $0(0,2)$ & $0(0,2)$ & -0.105 & 0.917 \\
\hline 3 postoperative days (at rest) & $0(0, I)$ & $0(0,1)$ & -0.387 & 0.699 \\
\hline 3 postoperative days (with motion) & $0(0,1)$ & $2(2,3)$ & -2.141 & 0.032 \\
\hline I postoperative month (at rest) & $0(0,1)$ & $0(0,1)$ & -1.009 & 0.313 \\
\hline I postoperative month (with motion) & $0(0,1)$ & $0(0,1)$ & -0.911 & 0.362 \\
\hline 3 postoperative months (at rest) & $0(0,0)$ & $0(0,1)$ & -1.270 & 0.204 \\
\hline 3 postoperative months (with motion) & $0(0,0)$ & $0(0,1)$ & -1.270 & 0.204 \\
\hline 6 postoperative months (at rest) & $0(0, I)$ & $0(0,1)$ & -1.192 & 0.233 \\
\hline 6 postoperative months (with motion) & $0(0,1)$ & $0(0,1)$ & -1.192 & 0.233 \\
\hline
\end{tabular}

Notes: The data are presented as median (interquartile range); there were no significant differences between groups, as determined by Mann-Whitney $U$-test ( $P>0.05$ ). Abbreviation: PACU, post-anesthesia care unit.

reported that $25.9 \%$ who underwent VATS developed postoperative neuropathic pain, and $18.8 \%$ reported pain that persisted one year postoperatively. ${ }^{2}$ Bayman et al. reported that incidence of chronic pain were similar after thoracotomy (33\%) and thoracoscopy (25\%). ${ }^{1}$ In our study, $25.6 \%$ of patients had chronic pain at 3 postoperative months, $30.6 \%$ of patients had chronic pain at 6 postoperative months. $12.9 \%$ experienced possible neuropathic pain and $2.6 \%$ experienced considerable neuropathic pain at 6 postoperative months. The incidence of chronic pain was similar with these above-mentioned studies, but incidence of neuropathic pain and average NRS was lower. This may be attributed to the well controlled acute postoperative pain. ${ }^{1}$

Recently, many hospitals have performed "enhanced recovery after surgery program," which uses different classes of analgesic drugs that are administered preoperatively and continued during the perioperative period. ${ }^{21}$ Both the groups in our study used multimodal analgesic plans with intraoperative opioids (sufentanil), weak 


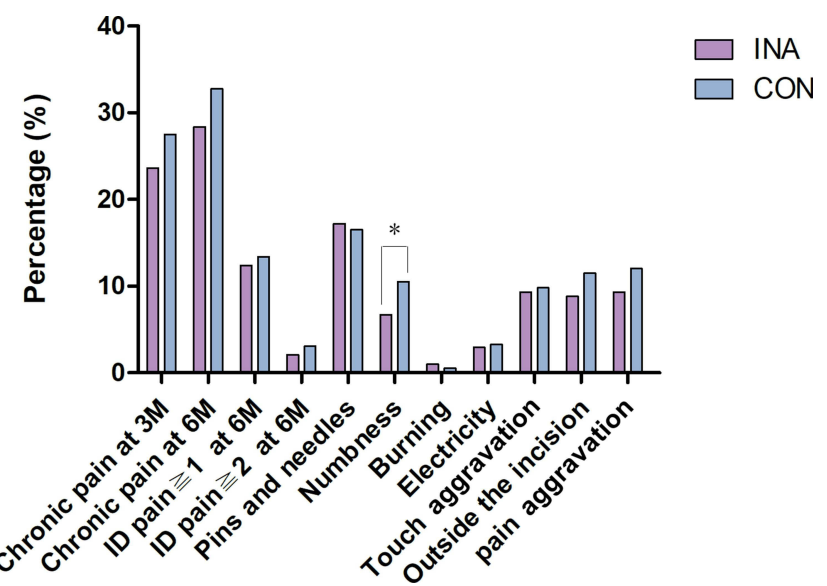

Figure 3 The data are presented as the numbers (\%), ${ }^{*} \mathrm{P}<0.05$ compared with the control group by the Chi square test.

opioids (dezocine), and nonsteroidal drugs (parecoxib). Postoperatively, we used oral drugs consisting of paracetamol and dihydrocodeine tartrate tablets (acetaminophen $500 \mathrm{mg}$ and dihydrocodeine tartrate $10 \mathrm{mg}$ ) $500 \mathrm{mg}$ three times and parecoxib $40 \mathrm{mg}$ two times in a day. Both groups had a low NRS during 6 postoperative months follow-up. The conventional analgesia group also had a low NRS score may due to multimodal analgesia.

Thoracic epidural analgesia is most effective for pain control and also for chronic pain in studies. ${ }^{6-8}$ However, the effects of regional anesthesia on chronic pain were unclear. Intercostal nerve blocks could relieve acute pain after VATS. ${ }^{22}$ In our study, the NRS score did not differ between the two groups. This may be due to injection only at the chest tube site but not incision site, and relatively weak pain after VATS might result in no sharp distinction with the addition of an intercostal blockade. Also, in order to get an exact analgesic effect, intercostal blockade was operated at the precise position under thoracoscopy without preemptive and preventive analgesia which was used in $\mathrm{CON}$ group. ${ }^{23}$

There are some limitations in this study. First, it was not a prospective, double-blinded, randomized trial. In our study, a total of 2012 patients were originally included, eventually 419 patients in the INA group and 418 in the CON group were analysed. Because of no significant difference in numbers and basic information of patients between the two groups, we did not construct a propensity analysis or match patients in each group. Selection bias may have existed in this research, and clinical data from electronic medical records may not be exact and is limited. Second, it often was difficult and not exact to assess pain by telephone. Third, the type of surgery and pathologies were not homogeneous. However, the similar situation of tumor size and lymph node dissection could guarantee undistorted results. Based on the above points, the conclusion may be less strongly stated. Thus, according to the results, we want to conduct a further study of RCT in order to improve the clinical analgesic regimen. Chronic post-thoracotomy pain remains an intractable problem, and future studies regarding different clinical analgesic regimens or new treatments are required.

\section{Conclusions}

In the context of multimodal analgesia, patients who underwent intermittent INA with $0.75 \%$ ropivacaine for VATS did not have an obvious effect on chronic postoperative pain.

Table 3 Postoperative Side Effects

\begin{tabular}{|c|c|c|c|c|}
\hline & $\begin{array}{l}\text { INA Group } \\
(N=419)\end{array}$ & $\begin{array}{c}\text { CON Group } \\
(N=418)\end{array}$ & $x^{2}$ & $P$-value \\
\hline Dizziness (n,\%) & $17(4.1)$ & $19(4.5)$ & 0.121 & 0.728 \\
\hline Nausea (n,\%) & $21(5.0)$ & $23(5.5)$ & 0.101 & 0.751 \\
\hline Vomiting $(n, \%)$ & $44(10.5)$ & $46(11.0)$ & 0.055 & 0.814 \\
\hline Blurred vision $(n, \%)$ & $0(0)$ & $0(0)$ & - & - \\
\hline Arrhythmia (n,\%) & $0(0)$ & $0(0)$ & - & - \\
\hline Dyspnea (n,\%) & $0(0)$ & $0(0)$ & - & - \\
\hline Epilepsy (n,\%) & $0(0)$ & $0(0)$ & - & - \\
\hline
\end{tabular}

Notes: The data are presented as the numbers (\%); there were no significant differences between groups, as determined by the Chi-square test $(P>0.05)$. 


\section{Acknowledgments}

We thank Yiducloud (Beijing) Technology Ltd for supporting part of the data extraction and processing. This study was supported by The Medical Science Research Foundation of Zhejiang Province (2019314366)

\section{Disclosure}

All authors declare no conflicts of interest.

\section{References}

1. Bayman EO, Parekh KR, Keech J, Selte A, Brennan TJ. A prospective study of chronic pain after thoracic surgery. Anesthesiology. 2017;126(5):938-951. doi:10.1097/ ALN.0000000000001576

2. Homma T, Doki Y, Yamamoto Y. Risk factors of neuropathic pain after thoracic surgery. J Thorac Dis. 2018;10(5):2898-2907. doi: $10.21037 /$ jtd.2018.05.25

3. Yekkirala AS, Roberson DP, Bean BP. Breaking barriers to novel analgesic drug development. Anesthesiology. 2017;126(5):938-951.

4. Joshi GP, Bonnet F, Shah R. A systematic review of randomized trials evaluating regional techniques for postthoracotomy analgesia. Anesth Analg. 2008;107(3):1026-1040. doi:10.1213/01.ane.0000333 274.63501.ff

5. Doi K, Nikai T, Sakura S, Saito Y. Intercostal nerve block with 5\% tetracaine for chronic pain syndromes. J Clin Anesth. 2002;14 (1):39-41. doi:10.1016/S0952-8180(01)00351-8

6. Lu YL, Wang XD, Lai RC. [Correlation of acute pain treatment to occurrence of chronic pain in tumor patients after thoracotomy]. Ai. Zheng $=$ Aizheng $=$ Chin J Cancer. 2008;27(2):206-209. [Chinese].

7. Ju H, Feng Y, Yang BX, Wang J. Comparison of epidural analgesia and intercostal nerve cryoanalgesia for post-thoracotomy pain control. Eur J Pain. 2008;12(3):378-384. doi:10.1016/j. ejpain.2007.07.011

8. Senturk M, Ozcan PE, Talu GK, Kiyan E. The effects of three different analgesia techniques on long-term postthoracotomy pain. Anesth Analg. 2002;94(1):11-15. doi:10.1213/00000539200201000-00003

9. Eguchi Y, Suzuki M, Sato T. Post-operative spinal epidural hematoma after thoracic and lumbar spinous process-splitting laminectomy for thoracic and lumbar spinal stenosis. Spine Surg Relat Res. 2019;3 (3):244-248. doi:10.22603/ssrr.2018-0086

10. Mimata R, Higashi M, Yasui M, Hirai T, Yamaura K. Spinal epidural hematoma following epidural catheter removal in a patient with postoperative urgent coronary intervention and intra-aortic balloon pumping (IABP): a case report. Am J Case Rep. 2019;20 (1):1356-1359. doi:10.12659/AJCR.917716
11. Ueda K, Hayashi M, Murakami J, Tanaka T, Utada K, Hamano K. Intercostal block vs epidural analgesia in thoracoscopic lung cancer surgery: a randomized trial. Gen Thorac Cardiovasc Surg. 2020;68 (3):254-260. doi:10.1007/s11748-019-01197-1

12. Weinstein EJ, Levene JL, Cohen MS, et al. Local anaesthetics and regional anaesthesia versus conventional analgesia for preventing persistent postoperative pain in adults and children (Review). Cochrane Database Syst Rev. 2018;6. doi:10.1002/14651858. CD007105.pub4

13. Jensen MP. Review of measures of neuropathic pain. Curr Pain Headache Rep. 2006;10(3):159-166. doi:10.1007/s11916-006-0041-z

14. Turkel Y, Turker H, Demir IA, Bayrak AO. Validation of self report version of the leeds assessment of neuropathic symptoms and signs score for identification of neuropathic pain in patients from northern Turkey. Adv Clin Exp Med. 2014;23(4):599-603. doi:10.17219/acem/ 37233

15. Li J, Feng Y, Han JS, Fan BF, Wang JS. Establishment and multicentre verification of three diagnostic scales for neuropathic pain in the Chinese version. Chin J Painol. 2011;17(9):549-553.

16. Hopkins KG, Hoffman LA, Dabbs Ade V. Postthoracotomy pain syndrome following surgery for lung cancer: symptoms and impact on quality of life. $J$ Adv Pract Oncol. 2015;6(2):121-132. doi:10.6004/jadpro.2015.6.2.4

17. Yang CC, Ro LS, Tsai YC. Development and validation of a Taiwan version of the ID pain questionnaire (ID Pain-T). J Chin Med Assoc. 2018;81(1):12-17. doi:10.1016/j.jcma.2017.06.019

18. Macrae WA. Chronic pain after surgery. Br J Anaesth. 2001;87 (1):88-98. doi:10.1093/bja/87.1.88

19. Rogers ML, Henderson L, Mahajan RP. Preliminary findings in the neurophysiological assessment of intercostal nerve injury during thoracotomy. Eur J Cardiothorac Surg. 2002;21(2):298-301. doi:10.1016/S1010-7940(01)01104-6

20. Inada K, Shirakusa T, Yoshinaga Y. The role of video-assisted thoracic surgery for the treatment of lung cancer: lung lobectomy by thoracoscopy versus the standard thoracotomy approach. Int Surg. 2000;85(1):6-12.

21. Piccioni F, Segat M, Falini S. Enhanced recovery pathways in thoracic surgery from Italian VATS Group: perioperative analgesia protocols. J Thorac Dis. 2018;10(4):S555-S563. doi:10.21037/ jtd.2017.12.86

22. Kelley TM, Bailey DW, Sparks P, Rice R, Caddell E. Intercostal nerve blockade with exparel $\AA^{\circledR}$ results in lower opioid usage during the first 24 hours after video-assisted thorascopic surgery. Am Surg. 2018;84(9):1433-1438. doi:10.1177/000313481808400945

23. Horn A, Kaneshiro K, Tsui BCH. Preemptive and preventive pain psychoeducation and its potential application as a multimodal perioperative pain control option: a systematic review. Anesth Analg. 2020;130(1):559-573. doi:10.1213/ANE.0000000000004319
Journal of Pain Research

\section{Publish your work in this journal}

The Journal of Pain Research is an international, peer reviewed, open access, online journal that welcomes laboratory and clinical findings in the fields of pain research and the prevention and management of pain. Original research, reviews, symposium reports, hypothesis formation and commentaries are all considered for publication. The manuscript management system is completely online and includes a very quick and fair peer-review system, which is all easy to use. Visit http:// www.dovepress.com/testimonials.php to read real quotes from published authors. 